\title{
Passive immunization to reduce Campylobacter jejuni colonization and transmission in broiler chickens
}

\author{
David Hermans ${ }^{1 *}$, Katleen Van Steendam², Elin Verbrugghe ${ }^{1}$, Marc Verlinden ${ }^{1}$, An Martel ${ }^{1}$, Tomasz Seliwiorstow ${ }^{3}$, \\ Marc Heyndrickx ${ }^{1,4}$, Freddy Haesebrouck' ${ }^{1}$ Lieven De Zutter ${ }^{3}$, Dieter Deforce ${ }^{2}$ and Frank Pasmans ${ }^{1}$
}

\begin{abstract}
Campylobacter jejuni is the most common cause of bacterium-mediated diarrheal disease in humans worldwide. Poultry products are considered the most important source of C. jejuni infections in humans but to date no effective strategy exists to eradicate this zoonotic pathogen from poultry production. Here, the potential use of passive immunization to reduce Campylobacter colonization in broiler chicks was examined. For this purpose, laying hens were immunized with either a whole-cell lysate or the hydrophobic protein fraction of $C$. jejuni and their eggs were collected. In vitro tests validated the induction of specific ImmunoglobulinY (IgY) against C. jejuni in the immunized hens' egg yolks, in particular. In seeder experiments, preventive administration of hyperimmune egg yolk significantly $(P<0.01)$ reduced bacterial counts of seeder animals three days after oral inoculation with approximately $10^{4} \mathrm{cfu} C$. jejuni, compared with control birds. Moreover, transmission to non-seeder birds was dramatically reduced (hydrophobic protein fraction) or even completely prevented (whole-cell lysate). Purified IgY promoted bacterial binding to chicken intestinal mucus, suggesting enhanced mucosal clearance in vivo. Western blot analysis in combination with mass spectrometry after two-dimensional gel-electrophoresis revealed immunodominant antigens of $C$. jejuni that are involved in a variety of cell functions, including chemotaxis and adhesion. Some of these (AtpA, EF-Tu, GroEL and CtpA) are highly conserved proteins and could be promising targets for the development of subunit vaccines.
\end{abstract}

\section{Introduction}

Today, campylobacteriosis is the most frequently reported zoonotic diarrheal disease worldwide, with Campylobacter jejuni as the major causative agent [1]. Despite many efforts aimed at minimizing Campylobacter contamination of poultry, no effective, reliable intervention measures exist to reduce bacterial numbers in the broiler gut [2]. In general, broiler chickens become colonized from the age of two weeks onward [3]. Transportation of immunoglobulin (Ig) Y, the major Ig class in chickens, from hen to embryo via egg yolk is believed to play a key role in the protection of young chicks with an immature immune system against Campylobacter colonization during two to three weeks post-hatch $[4,5]$. From two weeks onward, the concentration of

\footnotetext{
*Correspondence: david.hermans@nusciencegroup.com

'Department of Pathology, Bacteriology and Avian Diseases, Faculty of Veterinary Medicine, Ghent University, Salisburylaan 133, 9820 Merelbeke, Belgium

Full list of author information is available at the end of the article
}

maternally derived anti-Campylobacter IgY drops significantly, which coincides with an increased colonization susceptibility of the broiler chicks. Passive immunization of chicks may be prolonged by feeding broilers with high levels of anti-Campylobacter antibodies recovered from immunized hens [4]. Following immunization, specific IgY is induced and transferred from the serum to the egg yolk [4], where it is accumulated at high levels [6]. Indeed, preincubating $C$. jejuni with IgY from immunized hens has previously been shown to reduce fecal $C$. jejuni counts in broilers experimentally inoculated with this mixture [7]. In this study, also the effect of $\operatorname{IgY}$ on colonization in already-colonized animals was assessed. Results indicated that IgY induced only a limited therapeutic efficacy. After an initial drop, fecal $C$. jejuni numbers regained their original counts when treatment was stopped. Nevertheless, these observations indicate that IgY preparations from egg yolks could be a promising candidate to reduce $C$. jejuni colonization in broilers, but need to be optimized because

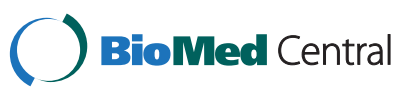


no studies regarding this topic were reported since. Moreover, in the study by Tsubokura et al. [7] fecal bacterial counts were determined, which may not perfectly correlate to determining cecal counts, which is more sensitive [8]. In the past two decades, some promising observations have been reported concerning the use of chicken egg yolk Igs in animal models and humans for the prevention and treatment of other bacterial and viral diseases, including streptococci [9], Helicobacter pylori [10], human rotavirus, enterotoxigenic Escherichia coli, Yersinia ruckeri and Salmonella species $[4,11]$. In these studies, protection was primarily observed after infection with the homologous strain.

Passive immunization using yolk IgY thus seems a promising strategy to control $C$. jejuni colonization in broiler flocks. For this purpose, the first aim of this study was to examine whether hyperimmune egg yolk could reduce or prevent cecal $C$. jejuni colonization in broiler chicks. The second objective was to identify the $C$. jejuni antigens reacting with $C$. jejuni-specific IgY raised in chicken eggs.

\section{Materials and methods}

\section{Experimental animals}

Commercial brown Leghorn laying hens and day-ofhatch Ross broiler chickens of both sexes from a local farm were raised in group until treatment. Birds were provided with a commercial feed and water ad libitum. Husbandry, euthanasia methods, experimental procedures and bio-safety precautions were approved by the Ethical Committee (EC) of the Faculty of Veterinary Medicine, Ghent University, Ghent, Belgium (EC numbers: 2010/174 and 2011/029). Chicks were examined for the presence of Campylobacter in mixed fecal samples using standard methods as described in [12] and proved to be free of Campylobacter.

\section{Bacterial strains and culture conditions}

C. jejuni strain $\mathrm{KC} 40$ from poultry origin was used for all experiments. This strain colonizes chickens to a high level $[12,13]$. Bacteria were routinely cultured in Nutrient Broth No.2 (NB2, CM0067; Oxoid Ltd., Basingstoke, Hampshire, UK) supplemented with Modified Preston Campylobacter-selective supplement (SR0204E; Oxoid) and Campylobacter-specific growth supplement (SR0232E; Oxoid), at $42{ }^{\circ} \mathrm{C}$ under microaerobic conditions $\left(5 \% \mathrm{O}_{2}, 5 \% \mathrm{CO}_{2}, 5 \% \mathrm{H}_{2}, 85 \% \mathrm{~N}_{2}\right)$. C. jejuni bacteria were enumerated by preparing 10-fold dilutions in Hank's Balanced Salt Solution (HBSS; GIBCO-BRL, Invitrogen, Carlsbad, CA, USA) and plating on modified charcoal cefoperazone deoxycholate agar (mCCDA, CM0739; Oxoid) supplemented with CCDA selective supplement (SR0155E; Oxoid) and Campylobacter-specific growth supplement, followed by microaerobic incubation at $42{ }^{\circ} \mathrm{C}$ for $22 \mathrm{~h}$.

\section{Extraction of $C$. jejuni hydrophilic and hydrophobic proteins}

To prepare the immunizing agent, cells from a $C$. jejuni culture were collected by centrifugation $(5000 \times g$ for 30 min at $4{ }^{\circ} \mathrm{C}$ ), washed in HBSS and sonicated on ice for $30 \mathrm{~s}$ in extraction buffer 1 (EB1; $40 \mathrm{~m} M$ Tris, supplemented with tributylphosphine solution, protease inhibitor cocktail, phosphatase inhibitors PP2 and PP3, DNase and RNase (Sigma-Aldrich, Steinheim, Germany) using a tip sonicator (Qsonica, Newtown, VS). After each sonication step, 12 in total, the sample was left on ice for $30 \mathrm{~s}$. After centrifugation at $16000 \times g$ for $10 \mathrm{~min}$ at $4{ }^{\circ} \mathrm{C}$ the supernatant (containing the hydrophilic proteins) was collected and the pellet resuspended in extraction buffer 2 (EB2; $40 \mathrm{~m} M$ Tris, supplemented with $5 M$ urea, $2 M$ thiourea, 2\% 3-[(3-cholamidopropyl)dimethylammonio]1-propanesulfonate (CHAPS), $0.2 \%$ carrier ampholytes, $100 \mathrm{~m} M$ dithiothreitol (DTT) and the respective inhibitors as mentioned above). After sonication and centrifugation of the latter suspension at $5000 \times g$ for $3 \mathrm{~min}$ to remove cell debris and non-lysed cells, the supernatant (containing the hydrophobic proteins) was either pooled with the first supernatant (to obtain the whole-cell protein lysate) or stored $\left(-20^{\circ} \mathrm{C}\right)$ separately.

\section{Immunization of laying hens}

Campylobacter-free commercial Brown Leghorn chickens were randomly assigned to vaccination groups at the age of 19 weeks. Chickens were immunized with either $50 \mu \mathrm{g}$ of the C. jejuni KC40 whole-cell lysate (EB1 + EB2) or $25 \mu \mathrm{g}$ protein of the hydrophobic protein fraction only (EB2), by intramuscular injection of $250 \mu \mathrm{L}$ of a 1:1 mixture with Freunds' Complete Adjuvant (FCA) at four different sites of the pectoral muscle. Chicks of the control group were immunized with a mixture of HBSS and FCA. Starting from the age of 21 weeks, three booster immunizations using Freunds' Incomplete Adjuvant (FIA) were given in a two-weekly time interval. Starting from one week after the second boost, eggs of the animals were collected and stored at $4{ }^{\circ} \mathrm{C}$.

\section{Determination of egg yolk and white $\lg \mathrm{Y}, \lg \mathrm{A}$ and $\lg \mathrm{M}$ titers}

At weekly time intervals, starting from the day of the first immunization, eggs were collected from both groups. The egg yolk and white were separated and pooled per group, diluted 1:5 (wt/vol) in distilled water and mixed thoroughly. After overnight incubation at $4{ }^{\circ} \mathrm{C}$, the supernatant, containing the water-soluble fraction of the egg yolk and white, respectively, was collected for immunoglobulin quantification using enzyme-linked immunosorbent assay (ELISA). Wells of a 
96-well Nunc-MaxiSorp microtiter plate were coated with $50 \mu \mathrm{L}$ of a suspension of C. jejuni KC40 cells $\left(2 \times 10^{7} \mathrm{cfu} /\right.$ $\mathrm{mL})$ in coating buffer $\left(2.16 \mathrm{~g} \mathrm{Na}_{2} \mathrm{CO}_{3} \cdot 10 \mathrm{H}_{2} \mathrm{O}+1.935 \mathrm{~g}\right.$ $\mathrm{NaHCO}_{3}$ in $500 \mathrm{~mL} \mathrm{H} \mathrm{H}_{2} \mathrm{O}$ ). Bacterial cells were either heat-killed or processed to antigenic fractions by sonication as described above. Plates were incubated overnight at $4{ }^{\circ} \mathrm{C}$, after which the wells were washed three times with HBSS followed by a final wash step using wash buffer (WB; PBS $+0.1 \%$ Tween 20). Next, the wells were incubated with $100 \mu \mathrm{L}$ blocking buffer (BB; WB $+1 \%$ bovine serum albumin) at room temperature (RT) for $1 \mathrm{~h}$ to reduce non-specific binding of antibodies. Subsequently, $100 \mu \mathrm{L}$ of two-fold dilutions (in BB) of the supernatant were added to the wells in triplicate. The plates were incubated at RT for $90 \mathrm{~min}$. After incubation, the wells were washed three times using HBSS and once using WB and $100 \mu \mathrm{L}$ of $1 / 10000$ (in WB) of horseradish peroxidase (HRP)-labelled anti-chicken IgY (Sigma), 1/20000 HRPlabelled goat anti-chicken IgA (Bio-Connect, The Netherlands) or 1/10000 HRP-labelled goat antichicken IgM (Gallus Immunotech Inc., Canada) were added to each well. After incubation and washing as described above, $50 \mu \mathrm{L}$ of $3,3^{\prime}, 5,5^{\prime}$-tetramethylbenzidine (TMB; Sigma) substrate was added. After $10 \mathrm{~min}$ the reaction was blocked by adding $50 \mu \mathrm{L} 0.5 \mathrm{M} \mathrm{H}_{2} \mathrm{SO}_{4}$ to the wells. The absorbance was then measured at $450 \mathrm{~nm}$ using an automated spectrophotometer. Antibody titres from egg yolks/whites of immunized hens were reported as the highest dilution where the optical density (OD) was greater than the $\mathrm{OD}+$ three standard deviations of wells containing yolk/white originating from birds that only received adjuvant.

\section{Purification of $\mathrm{lg}$ Y fraction from egg yolks}

IgY was purified from the egg yolks according to the method of Bird and Thorpe [14].

\section{Collection of broiler chicken intestinal mucus}

Commercial and Campylobacter-free 14-d-old broiler chicks were euthanized and the small intestine was collected and gently rinsed with phosphate-buffered saline (PBS) to remove fecal material. The mucus was scraped from the mucosa with a glass slide covered in parafilm, diluted 1:3 with N-2-hydroxyethylpiperazine-N'2-ethanesulfonic acid (HEPES, $25 \mathrm{mM}, \mathrm{pH}$ 7.4) and vortexed. The solution was centrifuged three times at $2000 \times g$ for $15 \mathrm{~min}$ at $4{ }^{\circ} \mathrm{C}$. The pooled supernatant containing the crude mucus was centrifuged two times more at $5000 \times g$ for $15 \mathrm{~min}$ at $4{ }^{\circ} \mathrm{C}$ and stored at $-80{ }^{\circ} \mathrm{C}$. Protein content was determined using a Coomassie (Bradford) Protein Assay Kit according to the manufacturer's instructions (Biorad, Nazareth, Belgium).

\section{Mucus adhesion test}

The effect of IgY on the adherence of C. jejuni to intestinal mucus was examined for the homolog C. jejuni strain $\mathrm{KC} 40$ as well as for five other $C$. jejuni strains (10kf-1.16, 7P6.12, 10C-6.1, 10kf-4.12 and 10VTDD-8). Bacteria were first incubated for $30 \mathrm{~min}$ at $42{ }^{\circ} \mathrm{C}$ in HBSS only or HBSS supplemented with purified IgY from C. jejuni-immunized or HBSS/sham-immunized hens. Mucus was diluted to a final concentration of $250 \mu \mathrm{g}$ protein $/ \mathrm{mL}$ in coating buffer. One hundred $\mu \mathrm{L}$ were immobilized per well of a microtiter plate (Maxisorp, Nunc) and incubated overnight at $4{ }^{\circ} \mathrm{C}$. The wells were washed three times with HBSS and saturated with $1 \%$ (wt/ vol) bovine serum albumin (BSA) in HBSS for $1 \mathrm{~h}$ at RT. After washing the wells two times with HBSS, $100 \mu \mathrm{L}$ of the $C$. jejuni-IgY mixture were transferred to the wells. After a $1 \mathrm{~h}$ incubation at $42{ }^{\circ} \mathrm{C}$, the wells were washed 15 times to remove unbound bacteria. Wells were thereafter treated with $200 \mu \mathrm{L} 0.5 \%$ (vol/vol) Triton X-100 to release adherent bacteria and incubated for $30 \mathrm{~min}$ at RT while shaking. Next, $300 \mu \mathrm{L}$ HBSS were added to each well and 10-fold dilutions of the wells were titrated on mCCDA plates.

\section{Motility assay}

To assess the influence of $C$. jejuni-specific IgY on $C$. jejuni motility of the six strains used in the study, bacteria were pre-incubated with purified IgY from yolks of either C. jejuni- or sham/HBSS-immunized laying hens and $20 \mu \mathrm{L}$ were pipetted onto semi-solid Mueller-Hinton $(\mathrm{MH})$ agar. After $24 \mathrm{~h}$ incubation at $42{ }^{\circ} \mathrm{C}$ under microaerobic conditions, the diameter of Campylobacter growth from both conditions was measured.

\section{Multilocus sequence typing}

To determine the relatedness between the $C$. jejuni strains used in this study, all strains were characterized by multilocus sequence typing (MLST) according to [15]. For determination of the sequence type (ST) and clonal complex (CC) all allelic sequences were queried against the online $C$. jejuni MLST database [16].

\section{Effect of in-feed hyperimmune egg yolk on transmission of and cecal colonization with $C$. jejuni in two-week-old broilers}

In trial 1, day-of-hatch Campylobacter free broiler chicks $(n=22)$ were raised in group. At 6 days of age the chicks were randomly assigned to 2 groups ( $n=11$ /group) and housed in separate isolating chambers. Animals of group 1 were provided with feed containing 5\% (wt/wt) egg yolk (mixed manually through the feed) from hens sham-immunized with HBSS/adjuvant, while birds of group 2 were fed 5\% (wt/wt) egg yolk from hens immunized with a whole-cell $C$. jejuni lysate/adjuvant mixture. 
Egg yolks were added to the feed for the remainder of the experiment. Equal amounts of feed and drinking water were provided for each group during treatment and care was taken that all animals had unlimited access to the feed and water. At the age of 10 days, three chicks of both groups were orally inoculated with approximately $8 \times 10^{3}$ cfu of $C$. jejuni strain KC40. At 13 days of age all animals were euthanized (as described above) and the ceca as well as their contents were collected for C. jejuni enumeration (see below).

In trial 2, day-of-hatch broiler chicks $(n=54)$ were raised in group. At 6 days of age the chicks were randomly assigned to 6 groups ( $n=9 /$ group) and housed in separate isolating chambers. Animals of groups 1,2 and 3 were provided with feed containing $5 \%$ (wt/wt) egg yolk from hens immunized with HBSS/adjuvant, while birds of groups 4, 5 and 6 were fed 5\% (wt/wt) egg yolk from hens immunized with the hydrophobic protein fraction of $C$. jejuni mixed with adjuvant. At the age of 10 days, three chicks of each group were orally inoculated with approximately $3 \times 10^{4} \mathrm{cfu}$ of $C$. jejuni strain KC40. At 13 days of age all animals were euthanized (as described above) and the ceca as well as their contents were collected for C. jejuni enumeration (see below).

\section{Cecal Campylobacter jejuni enumeration}

Ceca and contents were cut into small fragments, weighed, and diluted 1:9 (wt/vol) in NB2 with supplements. After homogenization, a 10 -fold dilution series was made in HBSS. Of each dilution, $100 \mu \mathrm{L}$ were spread on mCCDA plates. After $22 \mathrm{~h}$ incubation at $42{ }^{\circ} \mathrm{C}$ under microaerobic conditions, characteristic colonies were counted. For enrichment, diluted cecal samples in NB2 were incubated at $37{ }^{\circ} \mathrm{C}$ under microaerobic conditions. After $24 \mathrm{~h}$, samples were plated on mCCDA and incubated at $42{ }^{\circ} \mathrm{C}$ in a microaerobic environment. After 24 and $48 \mathrm{~h}$ plates were examined for the presence or absence of $C$. jejuni.

\section{Two-dimensional gel-electrophoresis and Western blot analysis}

To identify the immunodominant antigens of $C$. jejuni recognized by yolk IgY of immunized hens, the whole $C$. jejuni lysate was separated by iso-electric focussing (pH 3-10), followed by sodium dodecyl sulphatepolyacrylamide gel electrophoresis (SDS-PAGE) with a $4 \%$ stacking gel and $10 \%$ separating gel at $150 \mathrm{~V}$ for $30 \mathrm{~min}$ and $200 \mathrm{~V}$ for $60 \mathrm{~min}$ as previously described [17]. The separated proteins were transferred electrophoretically $(50 \mathrm{~V})$ onto nitrocellulose membranes (Bio-Rad) for $3 \mathrm{~h}$. After washing with milliQ water, the membrane blots were blocked in 5\% (wt/vol) milk in PBS for $1 \mathrm{~h}$ at RT. Subsequently, the blots were incubated overnight with purified egg yolk IgY, diluted to 1:5000 (vol/vol) in 5\% (wt/vol) milk. After washing with $0.05 \%$ (vol/vol) Tween-20, the blots were incubated with horseradish peroxidaseconjugated goat-anti-chicken immunoglobulin $\mathrm{G}$ at a dilution of 1:50 000 in $5 \%$ (wt/vol) milk for $1 \mathrm{~h}$. The blots were washed and proteins were immunodetected by enhanced chemiluminescence, using Supersignal West Dura Extended Duration Substrate (Pierce), and scanned and digitized using the Versa Doc imaging system. Blotting experiments were performed in duplicate to identify antigens recognized by IgY in yolk from immunized/ sham-immunized laying hens.

\section{In-gel digestion and mass spectrometric analysis}

Spots of interest (C. jejuni antigens reacting with hyperimmune egg yolk IgY) were cut out of the gel and processed as previously described [18]. Prior to mass spectrometry the isolated peptides were separated on a U3000 nano-HPLC device (Dionex) as previously described [19].

Identification of the peptides was performed on an electrospray ionization quadrupole time of flight (ESIQUAD-TOF) spectrometer (Ultima, Waters). Peptides were acquired in the range of 450 to $1650 \mathrm{~m} / \mathrm{z}$ in MS. The seven most abundant multiply charged ions with a minimum intensity of 60 counts per second were subjected to MS/MS (between 50 and $2300 \mathrm{~m} / \mathrm{z}$ ). m/z ratios selected for MS/MS were excluded for $150 \mathrm{~s}$.

Data analysis was performed against the Campylobacter protein databank (124864 sequences) from NCBI using the in-house search engine Mascot Daemon (2.3, Matrix Science, London, UK). Carbamidomethyl (N-term), deamidation (NQ) and oxidation (M) were set as variable modifications. Peptide mass tolerance and fragment mass tolerance were set at $0.35 \mathrm{Da}$ and $0.6 \mathrm{Da}$, respectively. Maximum two miss cleavages were allowed. Proteins were only considered to be positively matched if the significance was below $0.01(p \leq 0.01)$ and at least one peptide passing the required bold red criteria from Mascot Daemon, indicating that at least one peptide had rank 1 and a significance below 0.01 .

\section{Statistical analysis}

Data were analyzed by SPSS 17.0 software for Windows. The significance level $\alpha$ was set at 0.05. Campylobacter counts were first transformed to $\log _{10}$ counts before statistical analysis. For the in vivo trials, a nonparametric Mann-Whitney $U$ test was carried out to compare the means of $\log _{10}$ transformed counts in chicken cecal contents (of seeders, contact animals or both) of all groups (treated and control groups). For the mucus adhesion test, a one-way analysis of variance (ANOVA) was carried out to compare the means of $\log _{10}$ transformed adherent bacteria of all groups (treated groups and control groups). Significant differences were 


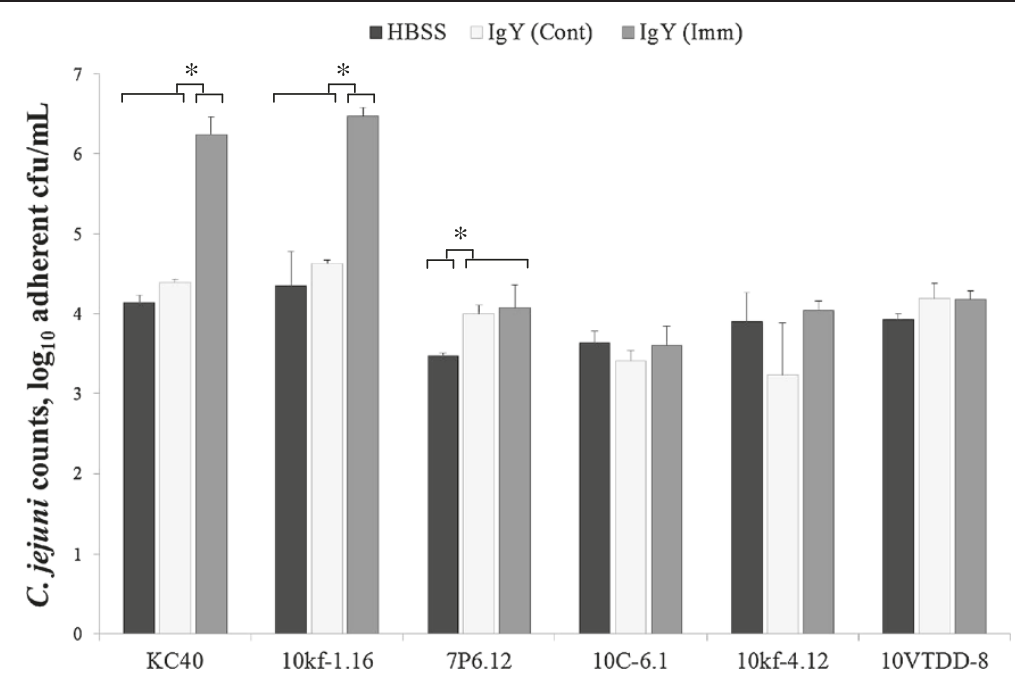

Figure 1 Campylobacter jejuni binding to chicken intestinal mucus. Total adherent Campylobacter jejuni bacteria to chicken intestinal mucus are shown after pre-incubation with HBSS (control, black rectangle symbol) or lgY derived from HBSS/sham-immunized (white rectangle symbol) or C. jejuni-immunized hens (grey rectangle symbol). Values are represented as $\log _{10}$ cfu adherent bacteria/well. Statistical differences are denoted with an asterisk $(p<0.05)$.

assessed by Bonferroni Post Hoc tests. P-values below 0.05 were considered significantly different.

\section{Results}

Determination of antibody titers from egg

C. jejuni-specific IgY were detectable in egg yolk of hens immunized with a $C$. jejuni lysate, resulting in titers of up to 1:16000 as determined by ELISA (results not shown). In contrast, $C$. jejuni-specific IgY in egg white and $C$. jejuni-specific IgA and IgM in egg white and yolk were not detectable after immunization.

\section{In vitro anti-Campylobacter properties of egg yolk IgY}

Pre-treatment with purified IgY from yolks of $C$. jejuni KC40-immunized hens significantly $(p<0.05)$ promoted bacterial binding of the homologous $C$. jejuni strain to chicken intestinal mucus by approximately $\log _{10} 2$ compared to IgY from HBSS/sham-immunized (control) animals (Figure 1). Increased bacterial binding to mucus after pre-treatment with KC40-derived IgY compared to control IgY was also observed for C. jejuni strain 10kf-1.16 $(p<0.05)$, whereas the binding capacity of the other four $C$. jejuni strains (7P6.12, 10C-6.1, 10kf4.12 and 10VTDD-8) remained unaltered $(p>0.05)$. All six $C$. jejuni strains tested belong to a different clonal complex, as proved by MLST analysis (Table 1), indicating that $C$. jejuni strain $\mathrm{KC} 40$ and $C$. jejuni strain $10 \mathrm{kf}-1.16$ are "true" heterologs. C. jejuni KC40-specific IgY did not affect $C$. jejuni motility of any of the tested strains (data not shown).

\section{In vivo anti-Campylobacter properties of egg yolk lgY}

In in vivo trial 1 , the overall cecal $C$. jejuni numbers (Figure 2) of inoculated (seeder) broilers receiving hyper-immune egg yolks from $C$. jejuni whole-cell lysate-immunized layers were reduced by $>5 \log _{10} \mathrm{cfu}$ $(p<0.01)$ compared to control seeders, receiving yolks from $\mathrm{HBSS} / \mathrm{sham}$-immunized hens $(3.3 \pm 1.2$ vs. $8.4 \pm 0.6$ $\log _{10} \mathrm{cfu} / \mathrm{g}$ cecal contents). Moreover, transmission to non-seeder (contact) chicks was completely prevented, in contrast to the control group where seeder and nonseeder birds $\left(8.4 \pm 0.6\right.$ vs. $7.3 \pm 1.1 \log _{10} \mathrm{cfu} / \mathrm{g}$ cecal

Table 1 Multilocus sequence type and clonal complex attribution of $C$. jejuni strains used in the study.

\begin{tabular}{|c|c|c|c|c|c|c|c|c|c|}
\hline \multirow[b]{2}{*}{ Isolate } & \multirow[b]{2}{*}{ Sequence type } & \multirow[b]{2}{*}{ Clonal complex } & \multicolumn{7}{|c|}{ MLST allelic profile } \\
\hline & & & aspA & $g \ln A$ & gltA & glyA & pgm & tkt & uncA \\
\hline KC40 & 794 & 677 & 10 & 81 & 50 & 87 & 120 & 76 & 52 \\
\hline $10 \mathrm{kf}-1.16$ & 267 & 283 & 4 & 7 & 40 & 4 & 42 & 51 & 1 \\
\hline 7P6.12 & 464 & 464 & 24 & 2 & 2 & 2 & 10 & 3 & 1 \\
\hline 10C-6.1 & 305 & 574 & 9 & 53 & 2 & 10 & 11 & 3 & 3 \\
\hline $10 \mathrm{kf}-4.12$ & 51 & 443 & 7 & 17 & 2 & 15 & 23 & 3 & 12 \\
\hline 10VTDD-8 & 905 & $N A^{1}$ & 2 & 15 & 4 & 3 & 154 & 25 & 35 \\
\hline
\end{tabular}

${ }^{1}$ not assigned. 


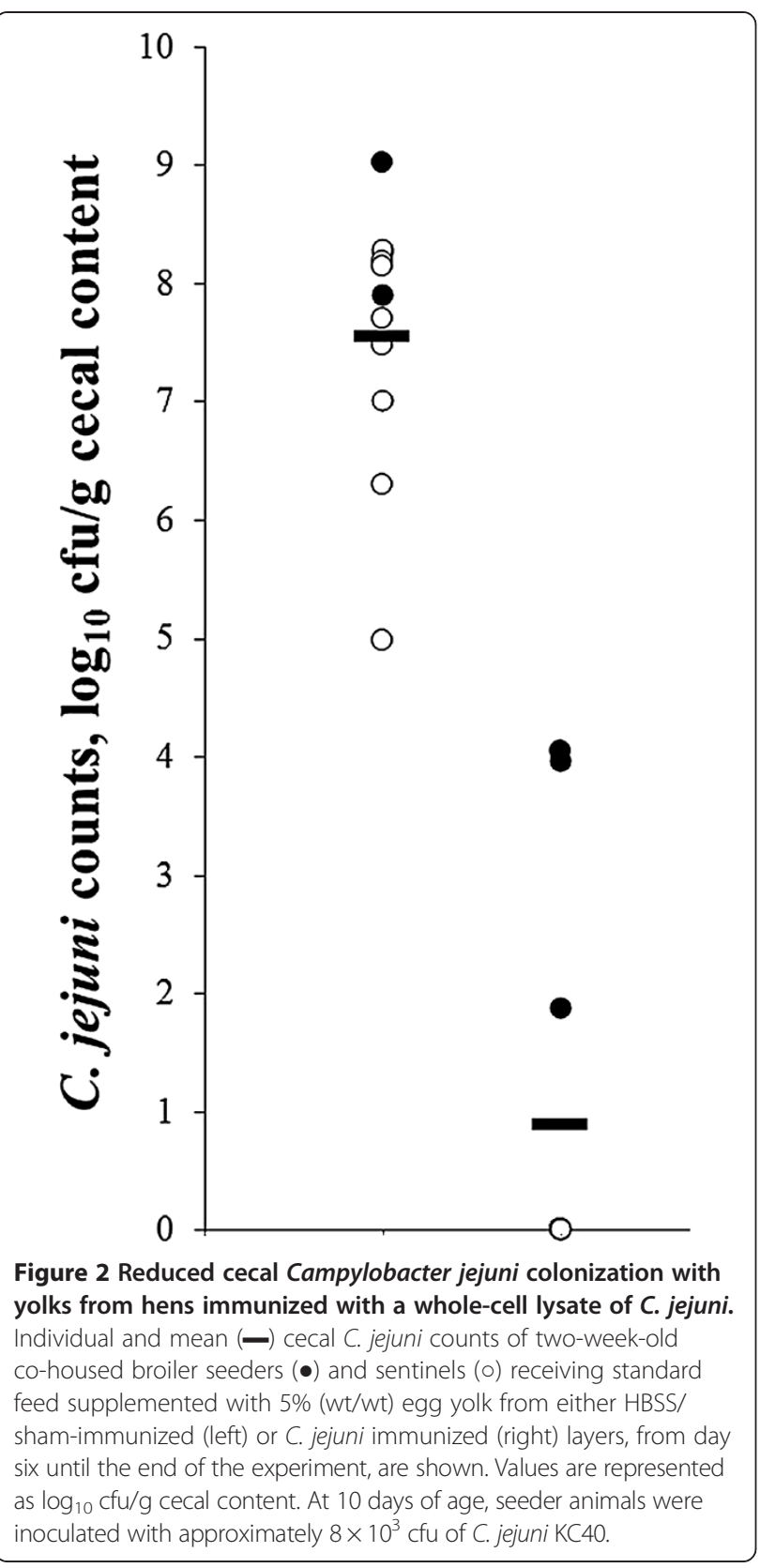

contents) were colonized to a similar $(p>0.05)$ degree. Together, this lead to a dramatic reduction $(p<0.001)$ in the overall cecal $C$. jejuni count by $\log _{10} 6.7$ for birds receiving hyperimmune yolk $\left(0.9 \pm 1.6 \log _{10} \mathrm{cfu} / \mathrm{g}\right.$ cecal contents) compared to birds receiving control yolks $\left(7.6 \pm 1.1 \log _{10} \mathrm{cfu} / \mathrm{g}\right.$ cecal contents).

In in vivo trial 2 , the protective effect of IgY induced against the outer-membrane proteins of $C$. jejuni was investigated. In two of the three replicate experiments (Figure $3 \mathrm{~A}$ and $\mathrm{B})$ a significant $(p \leq 0.05)$ reduction in cecal $C$. jejuni counts was observed in seeder animals (by respectively 3.3 and $1.6 \log _{10} \mathrm{cfu} / \mathrm{g}$ cecal contents) fed hyperimmune egg yolk compared to control birds. In the third replicate (Figure $3 C$ ), counts were reduced $(p>0.05)$ by $1.1 \log _{10} \mathrm{cfu}$. In replicate 1 , transmission to contact birds was completely prevented, while in replicate 2 and 3 cecal counts were significantly reduced by respectively 3.6 and $6.1(p<0.01) \log _{10}$ cfu/g cecal contents) when birds were fed hyperimmune egg yolk. Together, this resulted in an overall reduced cecal $C$. jejuni count in the replicate experiments of respectively $5.6(p=0.001), 2.9$ $(p=0.001)$ and $4.5(p<0.05) \log _{10} \mathrm{cfu} / \mathrm{g}$. Averaged over three replicates, cecal $C$. jejuni numbers of inoculated broilers receiving hyper-immune egg yolks from layers immunized with the hydrophobic protein fraction of C. jejuni were reduced by two $\log _{10}$ cfu $(p=0.002)$ compared to control animals $(6.0 \pm 1.8$ vs. $8.0 \pm 0.4$ $\log _{10} \mathrm{cfu} / \mathrm{g}$ cecal contents), while transmission to contact birds was greatly $(p<0.001)$ reduced $(1.5 \pm 2.5$ vs. $7.0 \pm 1.1 \log _{10} \mathrm{cfu} / \mathrm{g}$ cecal contents). Together, this lead to a dramatic reduction $(p<0.001)$ in the overall cecal $C$. jejuni count by $\log _{10} 4.4$ for broilers receiving hyperimmune yolk $\left(3.0 \pm 3.2 \log _{10} \mathrm{cfu} / \mathrm{g}\right.$ cecal contents) compared to birds receiving control yolks $\left(7.4 \pm 1.0 \log _{10}\right.$ $\mathrm{cfu} / \mathrm{g}$ cecal contents).

\section{Identification of immunodominant $C$. jejuni KC40 antigens} Western blots from 2-dimensional separated $C$. jejuni proteins immunostained with IgY from egg yolk of $C$. jejuni whole-cell lysate-immunized laying hens revealed several immunodominant antigens (Figure 4A), while blots stained with IgY from HBSS/sham-immunized hens showed only marginal reactivity (data not shown). Spots that were preferentially present on the Western blot immunostained with purified IgY from yolks of laying hens immunized with $C$. jejuni were linked to the 2-dimensionally separated $C$. jejuni proteins on the gel (Figure 4B), digested in peptides with trypsin and subjected to mass spectrometric (MS) analysis. Proteins with a Mascot score of $<56(p<0.01)$ or present in only one of duplicate experiments were excluded from the study. MS analysis identified several of the immunodominant antigens of $C$. jejuni KC40, represented in Table 2.

\section{Discussion}

Immunizing laying hens and subsequently collecting their eggs is a cheap and straightforward method to obtain high amounts of specific antibodies [6,11]. In our study, C. jejuni-specific IgY was dramatically induced in egg yolk of hens immunized with a $C$. jejuni whole-cell lysate, resulting in titers of up to 1:16 000 as determined by ELISA. In contrast, specific IgY in egg white and specific IgA and IgM in egg white and yolk were not significantly induced after immunization. This indicates that only transfer of IgY to egg yolk is biologically relevant in the overall transfer of immunoglobulins into eggs after 


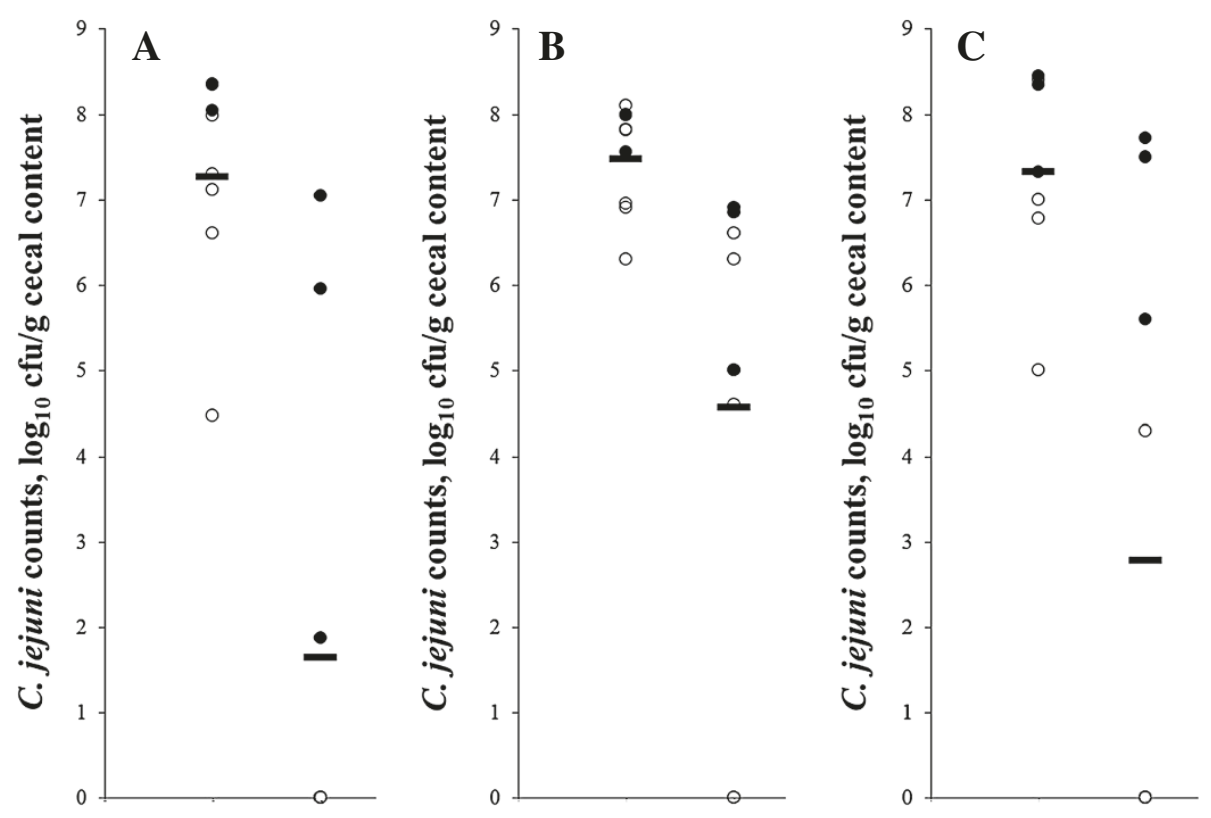

Figure 3 Reduced cecal Campylobacter jejuni colonization with yolks from hens immunized with C. jejuni hydrophobic proteins.

Individual and mean $(\boldsymbol{C}$ ) cecal C. jejuni counts of two-week-old co-housed broiler seeders $(\bullet)$ and sentinels $(0)$ receiving standard feed supplemented with $5 \%$ (wt/wt) egg yolk from either HBSS/sham-immunized (left) or C. jejuni immunized (right) layers, from day six until the end of the experiment, are shown. Values are represented as $\log _{10} \mathrm{cfu} / \mathrm{g}$ cecal content. At 10 days of age, seeder animals were inoculated with approximately $3 \times 10^{4} \mathrm{cfu}$ of $\mathrm{C}$. jejuni KC40. The experiment was performed in triplicate and results of all replicates are shown (A-C).

immunization of hens, which is in line with Dohms et al. [20].

In the first in vivo trial, using the whole-cell lysate of C. jejuni, broiler chicks were provided with feed supplemented with (hyperimmune) egg yolks from day seven onward. At 10 days of age three out of 11 birds were orally inoculated with approximately $8 \times 10^{3}$ cfu of $C$. jejuni. Colonization of these seeder animals is supposed to result in transmission of $C$. jejuni to the remainder of the birds. Three days after inoculation, cecal $C$. jejuni numbers of seeder birds receiving hyperimmune egg yolk were significantly reduced compared to control seeders without IgY (by over 5 logs). Moreover, none of the contact birds were colonized with $C$. jejuni, while chicks receiving control eggs carried high (ca. $\log _{10}$ $7 \mathrm{cfu} / \mathrm{g}$ ) bacterial numbers in their ceca. It is not clear at what site (the cecum or more proximal in the GI tract) the IgY fraction was active, but clearly it captured ingested $C$. jejuni bacteria, disabling them to colonize and transmit to other birds. In addition, specific IgY increased bacterial binding of $C$. jejuni to chicken intestinal mucus, suggesting that it promotes bacterial uptake in the mucus layer for enhanced mucosal clearance. Because several of the identified immunodominant antigens are believed to be associated with the bacterial outer-membrane, a second (analogous) in vivo trial was performed using egg yolks containing IgY against only the hydrophobic protein fraction of $C$. jejuni. Although the effects observed in this second in vivo trial were less pronounced compared to the results obtained in the first in vivo trial using IgY against the whole-cell lysate of C. jejuni (probably due to the higher inoculation dose of the seeders $\left(3 \times 10^{4} \mathrm{cfu}\right.$ vs. $\left.\left.8 \times 10^{3} \mathrm{cfu}\right)\right)$, hyperimmune yolks reduced cecal bacterial counts of seeders by 2 $\log _{10}$ cfu and dramatically reduced C. jejuni transmission to contact birds, indicating that the hydrophobic protein fraction is important for immunization but that also non-hydrophobic proteins may be involved in this immune response. Previous passive immunization studies with Salmonella enterica by other researchers [21] indicated no significant effect on cecal colonization of broilers receiving feed supplemented with freeze-dried egg yolk powder containing anti-Salmonella enterica IgY. Cecal Salmonella counts in birds receiving feed supplemented with $5 \%(\mathrm{wt} / \mathrm{wt})$ yolk powder on day 3 post-inoculation were, however, (non-significantly) reduced by three logs compared to control birds. These authors suggested that the antibodies were denatured and degraded along the GI tract, thereby reaching the ceca at insufficient concentrations, but it cannot be ruled out that a significant reduction could be achieved using more animals per group. In our study, egg yolks were administered as such to exclude degradation during freeze-drying. This might indicate that egg yolks form a protective matrix allowing IgY to survive the digestive enzymes and the low $\mathrm{pH}$ along the GI tract [11]. Other 


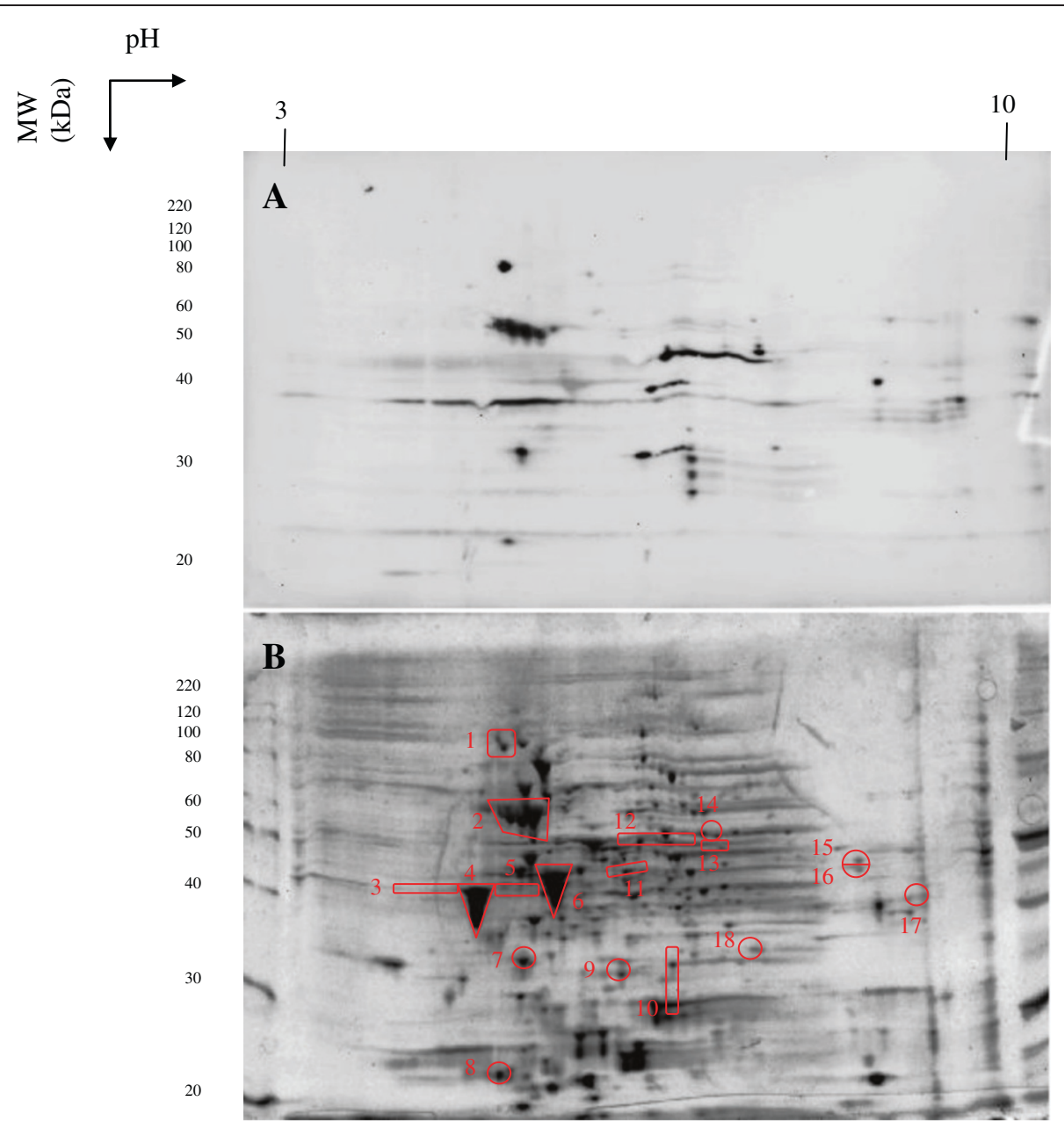

Figure 4 Western blot reacted with yolk immunoglobulin $\mathrm{Y}$ of hens immunized with a whole-cell C. jejuni lysate (A) and Campylobacter jejuni KC40 2D-proteome profile (B). A total protein extract of C. jejuni KC40 was separated by 2-dimensional gelelectrophoresis and separated proteins were detected by SYPRO ${ }^{\bullet} \mathrm{RUBY}$ Protein staining. Immunoreactive antigens are indicated with a number and were excised from the gel and sequenced using mass spectrometry. Identified proteins are indicated by the spot numbers given in Table 2.

factors that may be responsible for the differential outcome between our study and that of Chalghoumi et al. [21] include (1) age of the animals at challenge, (2) experimental set-up, since we used a seeder experiment while all birds were challenged with Salmonella, (3) immunization protocol and (4) bacterium-specific differences between Salmonella enterica and C. jejuni.

We identified the most likely $C$. jejuni proteins responsible for the induction of specific IgY. Because a $C$. jejuni whole-cell lysate was used, specific IgY could be induced against both membrane-bound as well as cytoplasmic proteins. Although our obtained results are fairly consistent with those reported in other studies examining antigenic proteins of $C$. jejuni, there was a striking absence in our study of Omp18, Cme, Cja and PEB proteins, identified in another study [22]. These differences might be in part explained by $C$. jejuni strain differences. In addition, it is not clear whether repeated immunization of chickens in their breast muscle could lead to a different immune response compared to that observed during natural gut colonization with $C$. jejuni.

A first group of proteins that reacted with the purified egg yolk IgY are flagellar proteins: flagellar hook protein FlgE, major flagellin FlaA and minor flagellin FlaB. FlgE and FlaA are known to be immunogenic and are needed for full motility of C. jejuni [22,23]. In addition, FlgE mediates flagellar assembly and protein secretion in C. jejuni [22], while FlaA mutation may result in reduced colonization in chicks [24]. These results are consistent with Shoaf-Sweeney et al. [22] who reported the presence of maternal antibodies reacting with these flagellar proteins in the serum of laying hens. Despite these flagellar proteins, which were shown to be highly immunogenic in this study, specific IgY did not affect C. jejuni motility. The major outer-membrane protein (MOMP) of $C$. jejuni, encoded by the porA gene, was identified in 
Table 2 Predicted immunodominant Campylobacter jejuni KC40 antigens identified with HPLC-MS/MS.

\begin{tabular}{|c|c|c|c|c|c|}
\hline Spot no. & Protein name & Gene & Mascot score & MW (kDa) & $\mathrm{pl}$ \\
\hline 1 & Flagellar hook protein & $f l g E$ & 1928 & 90.1 & 4.85 \\
\hline \multirow[t]{4}{*}{2} & Flagellin subunit protein A & flaA & 4958 & 59.2 & 5.7 \\
\hline & Co-chaperonin GroEL & groEL & 3281 & 58 & 5.02 \\
\hline & Flagellin subunit protein B & $f l a B$ & 1476 & 59.4 & 5.66 \\
\hline & Flagellin subunit protein B & $f l a B$ & 1048 & 26.3 & 5.52 \\
\hline \multirow[t]{2}{*}{3} & Flagellin subunit protein A & flaA & 693 & 58.8 & 5.53 \\
\hline & Co-chaperonin GroEL & groEL & 493 & 58 & 5.02 \\
\hline \multirow[t]{3}{*}{4} & Major outer membrane protein & omp & 3071 & 43.1 & 4.54 \\
\hline & Glutamate-1-semialdehyde-2,1-aminomutase & hemL & 356 & 46.5 & 8.92 \\
\hline & Glutathionylspermidine synthase family protein & & 164 & 44.8 & 4.59 \\
\hline 5 & Major outer membrane protein & omp & 1011 & 43.1 & 4.54 \\
\hline \multirow[t]{3}{*}{6} & Translation elongation factor thermo unstable & eF-Tu & 2172 & 43.6 & 5.11 \\
\hline & ATP-dependent Clp protease, ATP-binding subunit & $c l p X$ & 311 & 46.1 & 5.21 \\
\hline & Major outer membrane protein & omp & 66 & 36 & 4.53 \\
\hline \multirow[t]{4}{*}{7} & Chemotaxis protein $\mathrm{V}$ & chev & 1552 & 35.8 & 4.92 \\
\hline & Translation elongation factor thermo unstable & eF-Tu & 461 & 45 & 5.43 \\
\hline & Glycyl-tRNA synthetase alpha subunit & glyQ & 148 & 24.3 & 4.6 \\
\hline & Phosphoribosylformylglycinamidine cyclo-ligase & purM & 70 & 36 & 5.07 \\
\hline \multirow[t]{5}{*}{8} & FKBP-type peptidyl-prolyl cis-trans isomerase & slyD & 743 & 20.5 & 4.75 \\
\hline & Chemotaxis protein $\mathrm{V}$ & chev & 330 & 35.8 & 4.92 \\
\hline & Inorganic pyrophosphatase & ppa & 308 & 19.4 & 4.79 \\
\hline & Translation elongation factor thermo unstable & eF-Tu & 226 & 43.6 & 5.11 \\
\hline & Heat shock protein GrpE & grpE & 168 & 20.2 & 4.79 \\
\hline 9 & Translation elongation factor thermo unstable & eF-Tu & 96 & 43.6 & 5.11 \\
\hline \multirow[t]{3}{*}{10} & Branched-chain amino acid aminotransferase & $i l v E$ & 551 & 33.1 & 6.12 \\
\hline & Enoyl-(acyl-carrier-protein) reductase & $f a b l$ & 354 & 30 & 5.67 \\
\hline & Putative methyltransferase & & 174 & 29.8 & 5.92 \\
\hline \multirow[t]{4}{*}{11} & Methyl-accepting chemotaxis protein & & 811 & 40.6 & 5.5 \\
\hline & Succinyl-CoA synthetase beta chain & & 502 & 41.9 & 5.61 \\
\hline & Cysteine desulfurase, putative & & 472 & 43.2 & 5.83 \\
\hline & Phosphoglycerate kinase & $p g k$ & 216 & 43.9 & 6.07 \\
\hline \multirow[t]{7}{*}{12} & Conserved hypothetical protein & & 568 & 53 & 6.28 \\
\hline & Pyruvate kinase & pyk & 420 & 54 & 5.68 \\
\hline & Glutamyl-tRNA synthetase & $g \mid t X-1$ & 306 & 53.3 & 6.19 \\
\hline & ATP synthase F1, alpha subunit & $\operatorname{atpA}$ & 343 & 53.4 & 5.73 \\
\hline & Trigger factor & tig & 255 & 50.9 & 5.69 \\
\hline & Aspartate ammonia-lyase & aspA & 219 & 52.1 & 5.5 \\
\hline & Fumarate hydratase & fumc & 131 & 23.2 & 6.05 \\
\hline \multirow[t]{2}{*}{13} & ATP synthase F1, alpha subunit & $\operatorname{atp} A$ & 856 & 53.4 & 5.73 \\
\hline & GMP synthase (glutamine-hydrolyzing) & guaA & 580 & 57.4 & 6.19 \\
\hline \multirow[t]{3}{*}{14} & ATP synthase F1, alpha subunit & $\operatorname{atp} A$ & 1014 & 53.4 & 5.73 \\
\hline & Peptide transport system substrate-binding protein & & 488 & 58.7 & 6.52 \\
\hline & Acetyl-CoA carboxylase, biotin carboxylase & $\operatorname{accC}-2$ & 128 & 54.9 & 6.4 \\
\hline 15 & Putative secreted carboxyl-terminal protease & $\operatorname{ctp} A$ & 1607 & 48.3 & 8.7 \\
\hline
\end{tabular}


Table 2 Predicted immunodominant Campylobacter jejuni KC40 antigens identified with HPLC-MS/MS. (Continued)

\begin{tabular}{|c|c|c|c|c|c|}
\hline 16 & Putative secreted carboxyl-terminal protease & $\operatorname{ctp} A$ & 717 & 48.3 & 8.7 \\
\hline \multirow[t]{2}{*}{17} & $\begin{array}{l}\text { High affinity branched-chain amino acid ABC transporter, } \\
\text { periplasmic amino acid-binding protein }\end{array}$ & & 420 & 39.9 & 8.94 \\
\hline & Hydrogenase expression/formation protein & hypD & 151 & 40.9 & 8.59 \\
\hline \multirow[t]{3}{*}{18} & Dihydrodipicolinate synthase & $\operatorname{dapA}$ & 315 & 33 & 6.02 \\
\hline & Putative UDP-glucose 4-epimerase & & 214 & 35.6 & 7 \\
\hline & O-acetylserine sulfhydrylase B & cysM & 201 & 32.5 & 6.62 \\
\hline
\end{tabular}

several gel pieces, pointing to its abundance in the $C$. jejuni proteome [25]. In addition to being immunogenic [26], MOMP is involved in adhesion and transmembrane ion transport in C. jejuni [25]. However, MOMP is extremely genetically diverse and several conformational epitopes have been implicated in the induction of protective immunity [26], thereby possibly hindering its use in vaccine applications. Methyl-accepting chemotaxis (MCP) proteins are transmembrane receptors for chemotactic stimuli [23]. Attached to these transmembrane receptors is the $C$. jejuni chemotaxis protein $\mathrm{V}(\mathrm{CheV})$, a coupling protein involved in transducing chemotactic signals in C. jejuni [27], which was also identified in this study. Another immunodominant protein was the branched-chain amino acid ATP-binding cassette transport protein LivJ, a periplasmic binding protein probably involved in transporting amino acids into the bacterial cell [28] and crucial for chick colonization [22]. An additional role for LivJ in the interaction of C. jejuni with the chick cecum was suggested by Ribardo and Hendrixson [28]. ATP synthase is abundantly present and highly conserved among bacteria [29]. The protein is associated with the membrane where it performs its role in energy metabolism. In this study, the alpha subunit of ATP synthase F1 (AtpA) was found to be immunogenic in $C$. jejuni, as already reported for C. concisus [29].

Several of the identified antigens in this study were proteins mainly located in the bacterial cytoplasm where they perform their respective functions. Some of these antigens may nevertheless be interesting candidates for vaccine development. GroEL is an extremely conserved [30] $60-\mathrm{kDa}$ heat shock protein that plays a crucial role in the $C$. jejuni stress response and has previously been shown to be an immunodominant antigen of $C$. jejuni [31]. Although mainly located in the bacterial cytoplasm, in Salmonella Typhymurium (and other bacteria) GroEL is suggested to be expressed on the cell surface as well since it has been shown to mediate Salmonella adhesion [32]. The highly conserved translation elongation factor thermo unstable (EF-Tu) was found to be abundantly present in the C. jejuni whole-cell lysate, which is in line with observations in other bacteria [33]. Despite its cytoplasmic role during protein synthesis, EF-Tu was shown to be translocated to the surface in several bacteria, where it mediates adhesion and invasion of host cells [33]. Immunization of mice with Burkholderia pseudomallei EF-Tu resulted in a potent immune response that was partially protective against melioidosis. Finally, a putative secreted carboxyl-terminal protease of C. jejuni, CtpA, was identified. This protein has been shown to be highly prevalent and conserved in Burkholderia mallei, another Gram-negative bacterium [34]. The exact function, as well as prevalence and conservation of CtpA in C. jejuni is less understood, but the immunodominance of this protein indicates CtpA might be an interesting candidate for subunit vaccin development.

This study reports some promising observations, but further research is needed before a commercial product can be promoted. First of all, it needs to be established whether this protective effect is maintained over periods exceeding three days post-inoculation. Second, the immunization protocol and the yolk dose in the feed need to be optimized. Further research will also have to elucidate whether this strategy is capable of providing crossprotection against heterologous $C$. jejuni strains. The increased mucosal adherence observed for at least one of the heterologous C. jejuni strains tested in this study, suggests that (partial) cross-protection may be attained, but in vivo trials are necessary to proof this hypothesis. Eventually, this may lead to the development of a passive immunization strategy that can successfully control $C$. jejuni in chickens. If the relevant protective antigens of $C$. jejuni could be identified and studies regarding conservation and prevalence among $C$. jejuni strains are conducted, subunit vaccines able to target a wide range of $C$. jejuni strains could be developed and used for immunization of hens in order to prepare yolks for passive immunization applications.

To conclude, we here demonstrate for the first time that feeding broilers IgY-rich yolks from hens immunized with $C$. jejuni dramatically reduces both $C$. jejuni numbers in the ceca after challenge with the homologous strain and transmission to non-inoculated contact birds, providing a solid base for further research regarding passive immunization to control C. jejuni colonization in broiler flocks. 


\section{Competing interests}

The authors declare that they have no competing interests.

\section{Authors' contributions}

$\mathrm{DH}$ participated in the design of the study, performed in vivo and in vitro experiments, analysed data and drafted the manuscript. KVS performed LC-MS/MS analyses and coordinated the immunoproteomics experiments. EV assisted in the in vitro experiments. MV and AM participated in the coordination and analyses of the in vivo trials. TS and LDZ provided the heterologous C. jejuni strains. TS assisted in the MLST analysis. MH edited the manuscript. LDZ, FH and DD participated in the coordination of the study. FH and DD edited the manuscript. FP coordinated and participated in the design of the study, helped to interpret the results and edited the manuscript. All authors read and approved the final manuscript.

\section{Acknowledgements}

This work was financed by a grant of the Federal Public Service for Health, Food Chain Safety and Environment (FOD, Brussels, Belgium): project RT08/8CAMPOUL. We would like to thank Hanne Vereecke and Nathalie Van Rysselberghe for their excellent technical assistance during the in vivo trials.

\section{Author details}

${ }^{1}$ Department of Pathology, Bacteriology and Avian Diseases, Faculty of Veterinary Medicine, Ghent University, Salisburylaan 133, 9820 Merelbeke, Belgium. ${ }^{2}$ Laboratory for Pharmaceutical Biotechnology, Faculty of Pharmaceutical Sciences, Ghent University, Harelbekestraat 72, 9000 Ghent, Belgium. ${ }^{3}$ Department of Veterinary Public Health and Food Safety, Faculty of Veterinary Medicine, Ghent University, Salisburylaan 133, 9820 Merelbeke, Belgium. ${ }^{4}$ Institute for Agricultural and Fisheries Research, Technology and Food Unit, Brusselsesteenweg 370, 9090 Melle, Belgium.

Received: 23 June 2013 Accepted: 11 February 2014

Published: 4 March 2014

\section{References}

1. EFSA: The European Union summary report on trends and sources of zoonoses, zoonotic agents and food-borne outbreaks in 2009. EFSA J 2011, 9:2090.

2. Hermans D, Van Deun K, Messens W, Martel A, Van Immerseel F, Haesebrouck F, Rasschaert G, Heyndrickx M, Pasmans F: Campylobacter control in poultry by current intervention measures ineffective: urgent need for intensified fundamental research. Vet Microbio/ 2011, 152:219-228.

3. van Gerwe T, Miflin JK, Templeton JM, Bouma A, Wagenaar JA, JacobsReitsma WF, Stegeman A, Klinkenberg D: Quantifying transmission of Campylobacter jejuni in commercial broiler flocks. Appl Environ Microbiol 2009, 75:625-628.

4. Chalghoumi R: Hen egg yolk antibodies (IgY), production and use for passive immunization against bacterial enteric infections in chicken: a review. Biotechnol Agron Soc Environ 2009, 13:295-308.

5. Cawthraw SA, Newell DG: Investigation of the presence and protective effects of maternal antibodies against Campylobacter jejuni in chickens. Avian Dis 2010, 54:86-93.

6. Dias da Silva W, Tambourgi DV: IgY: a promising antibody for use in immunodiagnostics and immunotherapy. Vet Immunol Immunopathol 2010, 135:173-180.

7. Tsubokura K, Berndtson E, Bogstedt A, Kaijser B, Kim M, Ozeki M, Hammarstrom L: Oral administration of antibodies as prophylaxis and therapy in Campylobacter jejuni-infected chickens. Clin Expl Immunol 1997, 108:451-455.

8. Woldemariam E, Bouma A, Vernooij JC, Stegeman A: The sensitivity and specificity of fecal and cecal culture for the detection of Campylobacter in Dutch broiler flocks quantified by Bayesian analysis. Int J Food Microbiol 2008, 121:308-312.

9. Smith DJ, King WF, Godiska R: Passive transfer of immunoglobulin Y antibody to Streptococcus mutans glucan binding protein B can confer protection against experimental dental caries. Infect Immun 2001, 69:3135-3142

10. Shimamoto C, Tokioka S, Hirata I, Tani H, Ohishi H, Katsu K: Inhibition of Helicobacter pylori infection by orally administered yolk-derived anti-Helicobacter pylori antibody. Hepatogastroenterology 2002, 49:709-714.
11. Mine $Y$, Kovacs-Nolan J: Chicken egg yolk antibodies as therapeutics in enteric infectious disease: a review. J Med Food 2002, 5:159-169.

12. Hermans D, Martel A, Van Deun K, Van Immerseel F, Heyndrickx M, Haesebrouck F, Pasmans F: The cinnamon-oil ingredient trans-cinnamaldehyde fails to target Campylobacter jejuni strain KC40 in the broiler chicken cecum despite marked in vitro activity. J Food Prot 2011, 74:1729-1734.

13. Van Deun K, Pasmans F, Ducatelle R, Flahou B, Vissenberg K, Martel A, Van den Broeck W, Van Immerseel F, Haesebrouck F: Colonization strategy of Campylobacter jejuni results in persistent infection of the chicken gut. Vet Microbiol 2008, 130:285-297.

14. Bird CR, Thorpe R. Purification of immunoglobulin Y (lgY) from chicken eggs. In The Protein Protocols Handbook. Part VII. Edited by Walker JM. Totowa, NJ: Humana Press Inc:; 2002, 1009-1011.

15. Dingle KE, Colles FM, Wareing DRA, Ure R, Fox AJ, Bolton FE, Bootsma HJ, Willems RJL, Urwin R, Maiden MCJ: Multilocus sequence typing system for Campylobacter jejuni. J Clin Microbiol 2001, 39:14-23.

16. Campylobacter MLST homepage. [http://pubmlst.org/campylobacter]

17. Van Steendam K, Tilleman K, De Ceuleneer M, De Keyser F, Elewaut D, Deforce D: Citrullinated vimentin as an important antigen in immune complexes from synovial fluid of rheumatoid arthritis patients with antibodies against citrullinated proteins. Arthritis Res Ther 2010, 12:R132.

18. Cheung KJ, Tilleman K, Deforce D, Colle I, Van Vlierberghe H: The HCV serum proteome: a search for fibrosis protein markers. J Viral Hepat 2009, 16:418-429.

19. Van Steendam K, De Ceuleneer M, Dhaenens M, Van Hoofstat D, Deforce D: Mass spectrometry-based proteomics as a tool to identify biological matrices in forensic science. Int J Legal Med 2013, 127:287-298.

20. Dohms JE, Saif YM, Bacon WL: Metabolism and passive transfer of immunoglobulins in the turkey hen. Am J Vet Res 1978, 39:1472-1481.

21. Chalghoumi R, Marcq C, Théwis A, Portetelle D, Beckers Y: Effects of feed supplementation with specific hen egg yolk antibody (immunoglobulin $Y$ ) on Salmonella species cecal colonization and growth performances of challenged broiler chickens. Poult Sci 2009, 88:2081-2092.

22. Shoaf-Sweeney KD, Larson CL, Tang X, Konkel ME: Identification of Campylobacter jejuni proteins recognized by maternal antibodies of chickens. Appl Environ Microbiol 2008, 74:6867-6875.

23. Hermans D, Pasmans F, Heyndrickx M, Van Immerseel F, Martel A, Van Deun K, Haesebrouck F: A tolerogenic mucosal immune response leads to persistent Campylobacter jejuni colonization in the chicken gut. Crit Rev Microbio/ 2012, 38:17-29.

24. Wassenaar TM, Van der Zeijst BA, Ayling R, Newell DG: Colonization of chicks by motility mutants of Campylobacter jejuni demonstrates the importance of flagellin A expression. J Gen Microbiol 1993, 139:1171-1175.

25. Islam A, Raghupathy R, Albert MJ: Recombinant PorA, the major outer membrane protein of Campylobacter jejuni, provides heterologous protection in an adult mouse intestinal colonization model. Clin Vaccine Immunol 2010, 17:1666-1671.

26. Huang S, Sahin O, Zhang Q: Infection-induced antibodies against the major outer membrane protein of Campylobacter jejuni mainly recognize conformational epitopes. FEMS Microbiol Lett 2007, 272:137-143.

27. Lertsethtakarn P, Ottemann KM, Hendrixson DR: Motility and chemotaxis in Campylobacter and Helicobacter. Annu Rev Microbiol 2011, 65:389-410.

28. Ribardo DA, Hendrixson DR: Analysis of the LIV system of Campylobacter jejuni reveals alternative roles for LivJ and LivK in commensalism beyond branched-chain amino acid transport. J Bacterio/ 2011, 193:6233-6243.

29. Kovach Z, Kaakoush NO, Lamb S, Zhang L, Raftery MJ, Mitchell H: Immunoreactive proteins of Campylobacter concisus, an emergent intestinal pathogen. FEMS Immunol Med Microbiol 2011, 63:387-396.

30. Thies FL, Weishaupt A, Karch H, Hartung HP, Giegerich G: Cloning, sequencing and molecular analysis of the Campylobacter jejuni groESL bicistronic operon. Microbiology 1999, 145:89-98.

31. Zhang M, Meng F, Cao F, Qiao B, Liu G, Liu H, Zhou Y, Dong H, Gu Y, Xiao D, Zhang $Y$, Zhang J: Cloning, expression, and antigenicity of 14 proteins from Campylobacter jejuni. Foodborne Pathog Dis 2012, 9:706-712.

32. Tsugawa H, Ito H, Ohshima M, Okawa Y: Cell adherence-promoted activity of Plesiomonas shigelloides groEL. J Med Microbiol 2007, 56:23-29. 
33. Nieves W, Heang J, Asakrah S, HönerzuBentrup K, Roy CJ, Morici LA: Immunospecific responses to bacterial elongation factor Tu during Burkholderia infection and immunization. PloS One 2010, 5:e14361.

34. Bandara AB, DeShazre D, Inzana TJ, Sriranganathan N, Schurig GG, Boyle SM: A disruption of $\operatorname{ctpA}$ encoding carboxy-terminal protease attenuates Burkholderia mallei and induces partial protection in CD1 mice. Microb Pathog 2008, 45:207-216.

doi:10.1186/1297-9716-45-27

Cite this article as: Hermans et al:: Passive immunization to reduce Campylobacter jejuni colonization and transmission in broiler chickens. Veterinary Research 2014 45:27.

\section{Submit your next manuscript to BioMed Central and take full advantage of:}

- Convenient online submission

- Thorough peer review

- No space constraints or color figure charges

- Immediate publication on acceptance

- Inclusion in PubMed, CAS, Scopus and Google Scholar

- Research which is freely available for redistribution 